\title{
The Construction of the Animal Husbandry Information System Based on the Technology of Map Conflation
}

\author{
Yue Guo, Zhongbin Su ${ }^{*}$, Weizheng Shen, Zhipeng Guo and Qingming Kong \\ School of Electronic Engineering and Information Northeast Agricultural University \\ Harbin, 150030, China \\ 373718332@qq.com
}

\begin{abstract}
The research aims to merge the sections of geographic information distribution of the large-scale farms information monitoring system and the farming enterprises filing system which are under the Animal Husbandry Bureau in Heilongjiang Province as a Geographic Information System (GIS) that based on the map conflation technology of topological relation. Applying a variety of algorithms of points, lines, surfaces to this study, and using optimized "Spider code" and matching algorithm based on area overlay rate to solve the map database conflation problem of two different sources but consistent geographic target. It not only improves the map accuracy and consistency, but also adds new space characteristics, and updates attribute information which associated with dataset spatial characteristics. It makes the personnel at all levels in the Animal Husbandry Bureau in Heilongjiang Province realize information sharing. It is conductive to promote the work of staff at all levels and provide decision support of data to the Animal Husbandry Bureau in Heilongjiang Province.
\end{abstract}

Keywords: map conflation, topological relation, GIS, Animal Husbandry, decision

\section{Introduction}

In recent years, driven by market and policy, livestock and poultry breeding industry showed a trend of gradual growth. Using Heilongjiang Province as an example gradually increased the number of farms in dairy cattle, beef cattle and pigs, etc. But because farmers live dispersedly, which makes it difficult to manage. With the policy support of Heilongjiang Provincial Animal Husbandry Bureau, a variety of large-scale farms emerged, then livestock and poultry breeding industry in Heilongjiang Province gradually realizes the scale level [1-4]. Due to the demand of different level staffs, the large-scale farms information monitoring system and the farming enterprises filing system were built respectively in different years with the relevant regulation support of the Provincial Animal Husbandry Bureau. Among them, the large-scale farms information monitoring system has realized the information auditing of the users' qualification and production, as well as the supervision, inspection and auditing work of the farms. The farming enterprises filing system mainly records for farms and livestock farming community with livestock and poultry breeding codes, which can realize inspecting on farms and livestock farming community (qualified or unqualified) and the statistical functions. The above two systems include geographic information distribution, respectively, with different symbols in the corresponding map marked corresponding geographical location of farms. Users can visually monitor the detailed information of farms on the map.

The way of data collection, benchmark and projection, the degree of generalization, scale, purpose and the understanding of the real world application each were not identical when the 
above two systems obtained the geographical target of Heilongjiang repeatedly. It leads to the multiple expressions of the same geographical target in different datasets, and makes it difficult to share information between staffs at all levels in the Animal Husbandry Bureau. In order to improve the accuracy and consistency of geospatial data, adding new spatial entities, updating properties which combined with spatial characteristics of the dataset, saving the resource of the platform of GIS SERVER, and reducing the cost of system development this study aims to merge these two systems. The combined system is called Heilongjiang animal husbandry information system. The key is on the consolidation of geographic information systems' modules. This study use the map conflation technology of topological relation, that is applying a variety of algorithms of points, lines, surfaces to this study, which makes it more convenient and direct for the staffs at all levels in the Animal Husbandry Bureau to manage all types of large-scale farms. It also provides decision support of data to the Animal Husbandry Bureau in Heilongjiang Province.

\section{The System Design}

\subsection{The Whole Frame for Heilongjiang Animal Husbandry Information System}

Combined with the $\mathrm{C}$ \# language, based on SOA architecture, Web application part of this system uses .Net as the developing platform. The section of geographic information adopts the series products of ArcGIS of the ESRI Company, and combines the secondary system development with $\mathrm{C}$ \# language. Meanwhile, it adopts the idea of object-oriented development, the three-tier architecture system and role-based permissions dynamic allocation technology.

At the same time, the system adopts integrated design of B / S (Browser / Server) and C / S (Client / Server). Based on the same data sources, this system provides a shared data channel. Two kinds of structure use the same data server and play their own technical advantages in different applications, which greatly enhance the overall advantages of the system [5-6]. Among them, users at all levels in province, city, county, township and village can access to the web server by a browser or a mobile terminal. Based on the different requests, the web server will further access to the basic information database or the map database. The architecture diagram of this system is shown in Figure 1.

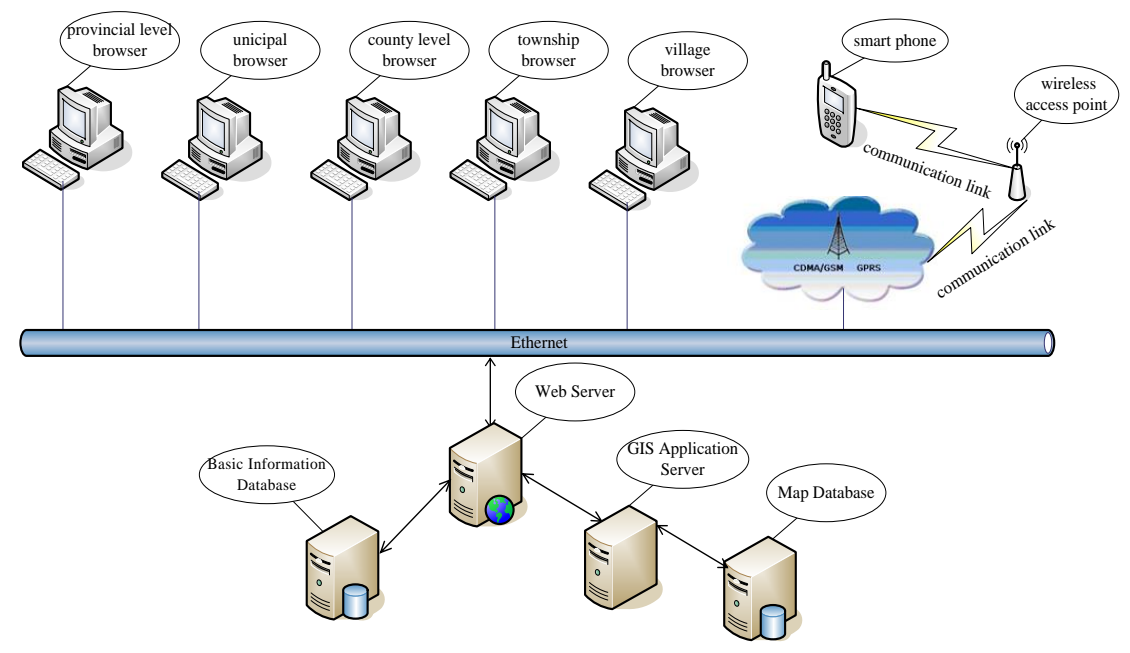

Figure 1. The System Architecture Diagram 


\subsection{The Function Modules of Heilongjiang Animal Husbandry Information System}

This system consists of two parts. The first part is the large-scale farms information monitoring system, and second part is the farming enterprises filing system. Among them, the large-scale farms information monitoring system contains business information management module and geographic information distribution module. The farming enterprises filing system contains business information management module and geographic information distribution module. The function module of this system is shown in Figure 2.

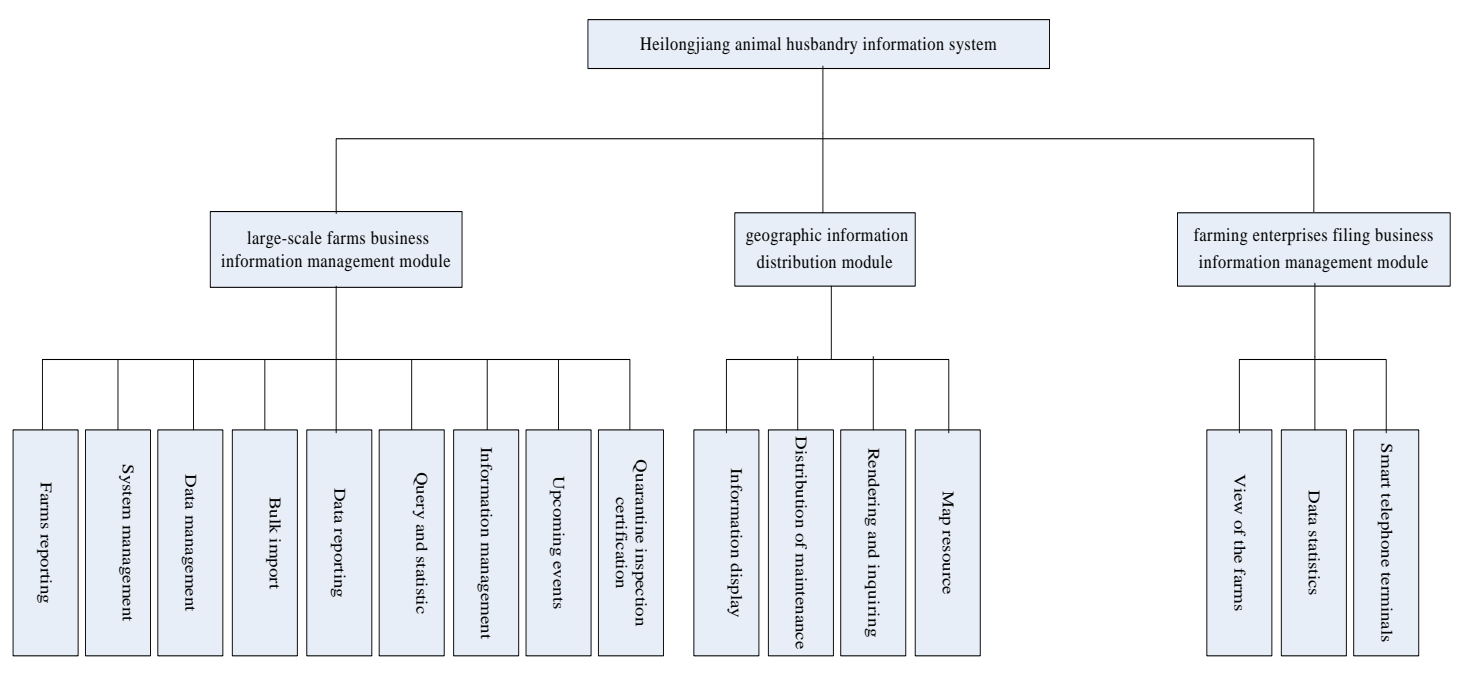

Figure 2. The Function Module of this System

\section{The Algorithm and Application for the Technology of Map Conflation}

The technology of map conflation is to build local coordinate transformation relationship between two or more map databases after matching the corresponding entity, so as to obtain fusion of graphic and attribute. It realizes the integration of the map database and fusion of information which is the same landmark but from different source [7].

The research adopts the technology of map conflation which is based on topological relation, merging the map databases of the large-scale farms information monitoring system and the farming enterprises filing system together. Map consolidation includes the following four steps. Firstly, pre-treat the data. Secondly, match the corresponding entities. Thirdly, adjust and merge the graphic data. Fourthly, transform or merge the attribute [8]. The following describes the process of consolidation of map databases of the large-scale farms information monitoring system and the farming enterprises filing system.

\subsection{The Pretreatment of Data}

In this process, it mainly involves the unification of data specifications, storage formats and the similar type of entities, the consistent transformation of data model and spatial reference on format, scale, Multi-Projection types and the geographical spatial data of geodetic coordinate system about the map databases of the large-scale farms information monitoring system and the farming enterprises filing system. Establish topological relation and set index for the spatial entity. 


\subsection{The Matching of Corresponding Entities}

Judge the degree of the difference between entities according to the spatial information and attribute information of entity. Then match corresponding entity by different algorithms in different situations according point, lines and surfaces, which in addition not only to solve the matching of one-to-one corresponding entities, but also solve the matching of one-to-many, and many-to-one and many-to-many corresponding entities [9]. The algorithm on the matching of corresponding entities will be introduced in three aspects according to the point, the lines and the surface.

Point matching refers to the process that identifies the same geographic and the same feature point in the real world reflected by two types of datasets. Saalfeld referred that two aspects should be considered in the matching of corresponding entities: The first is the number of lines connected to the point, which is the degree of that point. The second is the direction of the line which connected to the point. It means that using the distance between points to determine the candidate matching set first, then use some degrees and spider code to find the most similar point as the matching point [10]. But there are certain drawbacks in this algorithm. This design quotes the optimized algorithm in the eleventh literature to match the corresponding points. The optimized algorithm shows that take the node as origin to establish the coordinates, and take the positive direction of vertical axis as the initial direction, then divide the possible direction angle of the connection into eight equal areas which are consecutive disjoint. Then use a 16-bit encoding to represent the structure feature of a node, and each two bit correspond an area. Then give the two bits' encoding to the corresponding area in which the connections was left. If there is one connection of the point, the corresponding encoding is 01 . If there are two connections of the point, the corresponding encoding is 11 . If the connection left on the border of the area, the adjacent areas' encoding is 10 , or the encoding shows 00 [11].

Take the intersection point on the map of Shengye, Shengqin and Shengqian in Chaoyang of Shuangcheng as example, the intersection point in the map of the large-scale farms information monitoring system called $A$, and the intersection point in the map of the farming enterprises filing system called $A^{\prime}$. Point entity encoding schemes are shown as $a$ and $b$ in Figure 3. Take $A$ and $A^{\prime}$ as origin to establish the coordinates respectively, then divide it into eight equal areas. Then the results show that the encoding of $A$ is 0001000100000100 , and the encoding of $A^{\prime}$ is 0001000100000100.

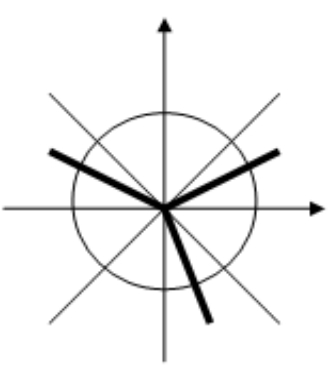

a. $A$

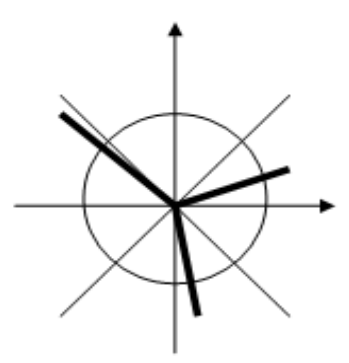

b. A

\section{Figure 3. Point Entity Encoding Schemes}

So the comparison of the same point can be converted to the calculation of the similarity of every encoding of the nodes. The calculation of the similarity value adopts 2 groups of encoding to do the "and" operation, then add up the number of 1 in the result which we name 
it as $N$ now, then $N$ divided by the minimum value of the two degrees of the nodes (The maximum value of $N$ may equal to the minimum value of the two degrees of the nodes) which result in the similarity of the two nodes. The result of the "and" calculation for $A$ and $A^{\prime}$ is 0001000100000100 , then $N$ equals to 3. Because the two degrees of $A$ and $A^{\prime}$ all equals to 3 , then the similarity of $A$ and $A^{\prime}$ equals to 1 . The threshold for the similarity of topology is sited as 0.70 in this research. Due to the value of the similarity of $A$ and $A^{\prime}$ is greater than the sited threshold. $A$ and $A^{\prime}$ matches.

Take farms and the surrounding feature as point entity, and follow the rules of Xi'an 80 . Take 10 corresponding nodes' coordinates of the projection of the same geographic goals which are the towns called Xingfu and Shuangcheng in Shuangcheng on the maps of the large-scale farms information monitoring system and the farming enterprises filing system respectively. The coordinates are shown in Table 1.

Table 1. Coordinates of the Projection of Farms and the Surrounding Feature

\begin{tabular}{cccccc}
\hline ID & $X$ & $Y$ & ID & $X$ & $Y$ \\
\hline 1 & 529261.939 & 5034845.831 & 1 & 529261.931 & 5034845.825 \\
2 & 530305.884 & 5037538.110 & 2, & 530305.876 & 5037538.104 \\
3 & 529921.273 & 5031659.051 & 3 & 529921.265 & 5031659.045 \\
4 & 532778.386 & 5030505.217 & 4, & 532778.379 & 5030505.212 \\
5 & 529976.217 & 5030834.884 & 5, & 529976.210 & 5030834.879 \\
6 & 526130.104 & 5030010.717 & 6 & 526130.095 & 5030010.712 \\
7 & 526185.049 & 5021823.991 & 7 & 526185.041 & 5021823.985 \\
8 & 522888.380 & 5035834.831 & 8, & 522888.377 & 5035834.825 \\
9 & 531624.552 & 5030175.550 & 9 & 531624.545 & 5030175.545 \\
10 & 531184.996 & 5030582.055 & & & \\
\hline
\end{tabular}

Take the points on the map of the large-scale farms information monitoring system as the matching points, and the points on the map of the farming enterprises filing system as the points to be adjusted. We can obtain the combined result which is based on the optimization algorithm referred above. The coordinates are shown in Table 2.

Table 2. The Combined Result Which is based on the Optimization Algorithm

\begin{tabular}{cc}
\hline Before adjustment & After adjustment \\
\hline$\left(1,1^{\prime}\right)$ & $(529261.939,5034845.831)$ \\
$\left(2,2^{\prime}\right)$ & $(530305.884,5037538.110)$ \\
$\left(3,3^{\prime}\right)$ & $(529921.273,5031659.051)$ \\
$\left(4,4^{\prime}\right)$ & $(532778.386,5030505.217)$ \\
$\left(5,5^{\prime}\right)$ & $(529976.217,5030834.884)$ \\
$\left(6,6^{\prime}\right)$ & $(526130.104,5030010.717)$ \\
$\left(7,7^{\prime}\right)$ & $(526185.049,5021823.991)$ \\
$\left(8,8^{\prime}\right)$ & $(522888.380,5035834.831)$ \\
$\left(9,9^{\prime}\right)$ & $(531624.552,5030175.550)$ \\
$(10)$ & $(531184.996,5030582.055)$ \\
\hline
\end{tabular}


Because the line entity is made up of some point entities, the line matching can be converted to the point matching. As long as they meet the following conditions, all of them can be matched. First of all, they are the entity of the same name. What's more, the outset and the destination can be matched. At last, the shape of the line is similar.

There are a variety of algorithms for the surface matching. Such as matching algorithm for the geometric distance between surface centroids, matching algorithm for the fuzzy topological relationships between surfaces and matching algorithm for surface of the function of steering angle [12]. This research adopts matching algorithm for the fuzzy topological relationships between surfaces. As a result of the split rate, Diego rate, degree of adhesion and degree of center are the main elements which measure the topological relationship between surface entities, and the definition of area Diego rate is the proportion of the area of two surface domain overlapping part and their own area. Area Diego rate is the most indicators reflecting surface topology relationship. So we adopt area Diego rate to calculate the same surface entity matching.

The following steps are matching algorithms that based on area Diego rate of the entity of the same name. If $A_{1}$ and $A_{2}$ is the surface entities to be adjusted in the spatial dataset of the map database of the large-scale farms information monitoring system and the farming enterprises filing system. And calculate their area Diego rate respectively.

$\operatorname{ove}\left(A_{1}, A_{2}\right)=\operatorname{Area}\left(A_{1}, A_{2}\right) / \operatorname{Area}\left(A_{1}\right)$

$\operatorname{ove}\left(A_{2}, A_{1}\right)=\operatorname{Area}\left(A_{1}, A_{2}\right) / \operatorname{Area}\left(A_{2}\right)$

If formula (1) and formula (2) are greater than the setting threshold at the same time, then $A_{1}$ and $A_{2}$ are the entities of the same name. If formula (1) and formula (2) are less than the setting threshold at the same time, then $A_{1}$ and $A_{2}$ are not the entities of the same name. If one of them is larger than the setting threshold and the other is less than the setting threshold, they can be judged as partial matching with the relationship of containing. That is, the situations of not one to one are existed. It is necessary to do the secondary matching between the collections of the surface entity to determine the matching relationship.

Take Fuzhi in Lequn of Shuangcheng as an example in the following. Merge the corresponding surfaces in the map of the large-scale farms information monitoring system and the farming enterprises filing system, and take the surface in the map of the large-scale farms information monitoring system as the matching entity named $A_{1}$. At the same time, take the surface in the map of the farming enterprises filing system as the entity to be adjusted

$$
\begin{aligned}
& \operatorname{Area}\left(A_{1}, A_{2}\right)=3.798 \quad, \quad \operatorname{Area}\left(A_{1}\right)=3.823 \quad \text { and } \operatorname{Area}\left(A_{2}\right)=3.819 \quad \text {,so } \\
& \text { ove }\left(A_{1}, A_{2}\right)=\operatorname{Area}\left(A_{1}, A_{2}\right) / \operatorname{Area}\left(A_{1}\right)=0.993 \\
& \text { ove }\left(A_{2}, A_{1}\right)=\operatorname{Area}\left(A_{1}, A_{2}\right) / \operatorname{Area}\left(A_{2}\right)=0.994
\end{aligned}
$$

We set the threshold of area Diego rate as 0.812 in this research. Because ove $\left(A_{1}, A_{2}\right)$ and ove $\left(A_{2}, A_{1}\right)$ are larger than the setting threshold, $A_{1}$ and $A_{2}$ are the matching entities of the same name. So taking $A_{1}$ as the matching surface, and taking $A_{2}$ as the surface to be adjusted, we can merge them successfully. The rendering that are matched successfully is shown as $b$ in Figure 4. 


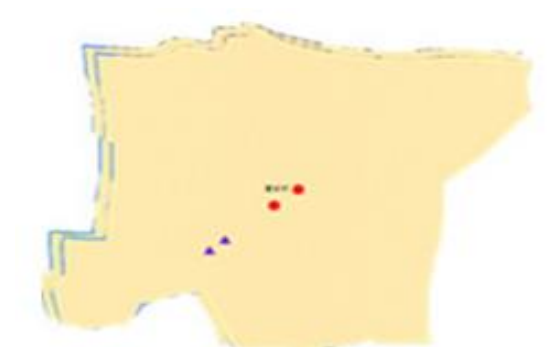

$a$. The rendering before merge them together

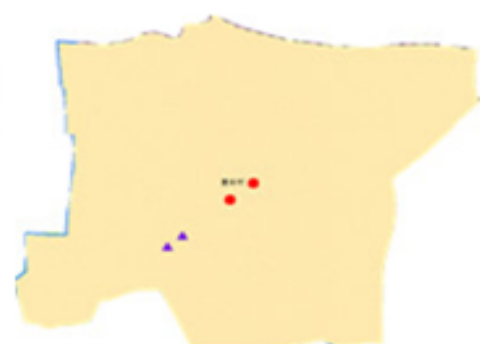

$b$. The rendering that are matched successfully

Figure 4. The Comparison of the Surface before Merging and After Merging

\subsection{Adjust and Merge the Graphic Data}

Because of many uncertain factors, there must be some entities being matched unsuccessfully in the three matching algorithm above. The research adopts the adjusting and merging algorithm which is based on the topological relationship to calculate the spatial locations which were not matched. The algorithm is shown in the following. First, the various entities are decomposed into some point entities, and then take the point which was matched unsuccessfully as the point to be adjusted, and take the point which was matched successfully as the matching point. Using breadth-first search algorithm to set off from every adjusted point, search and record all the matching point that connected with them and the length between the adjusted point and its matching point [13-15]. The threshold of the distance is sited as 0.16 meter in this research. Taking the length of path as weights, namely, the influence of longer distance is smaller, but the influence of shorter distance is bigger. To any point to be adjusted names as $\mathrm{P}$, the adjustment of coordinates can be determined by $Q_{i}(\mathrm{i}=1, \ldots \mathrm{N}) \quad N$ points, and the journey from $P$ to $Q_{i}$ is $L_{i}(\mathrm{i}=1, \ldots \mathrm{N})$, the amount of coordinates' adjustment for $Q_{i}$ is $\left(\Delta \mathrm{X}_{\mathrm{Q}_{\mathrm{i}}}, \Delta Y_{Q_{I}}\right)$, and then the amount of coordinates' adjustment for $P$ can be determined by (3).

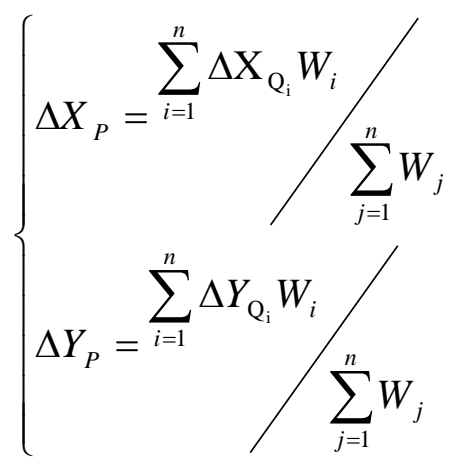

And the weights of formula (3) is $W_{i}=1 / L_{i}$. Based on the algorithms above, match the corresponding entities in all domains, and update the map database.

Then convert the corresponding data of attribute uniformly and merge them, which realize the sharing information. 


\section{The Realization of Algorithms and Functions}

\subsection{The Realization of Algorithms}

Based on the steps in part three, the map database of the large-scale farms information monitoring system and the farming enterprises filing system were merged successfully. All kinds of farms and farming communities in Heilongjiang were marked on the map. Among them, the red points represent the farms or farming communities of pig; the green squares represent the farms or farming communities of beef cattle; the purple triangles represent the farms or farming communities of cow. We can call the location of farms or farming communities which have been recorded or the location of all the farms or farming communities by corresponding code. The result of conflation is shown as $a, b$ and $c$ in Figure 5.

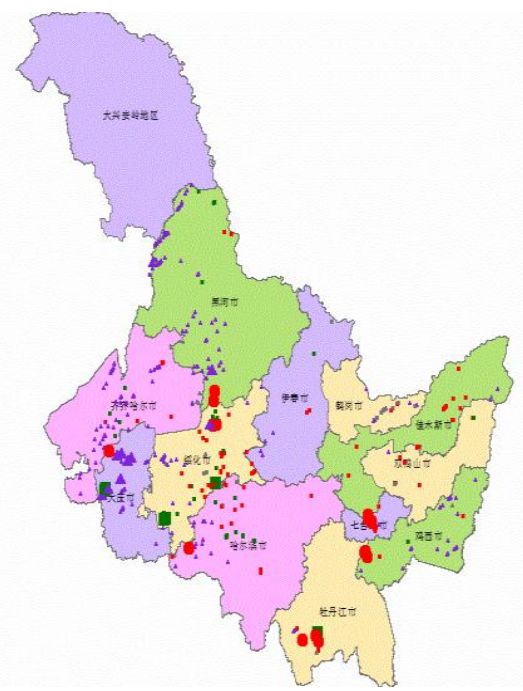

$a$.The map of the farming enterprises filing system

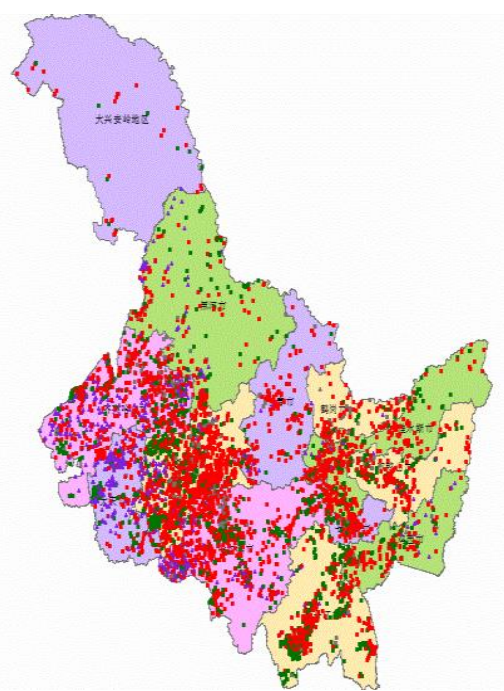

$b$. The map of the large-scale farms information monitoring system

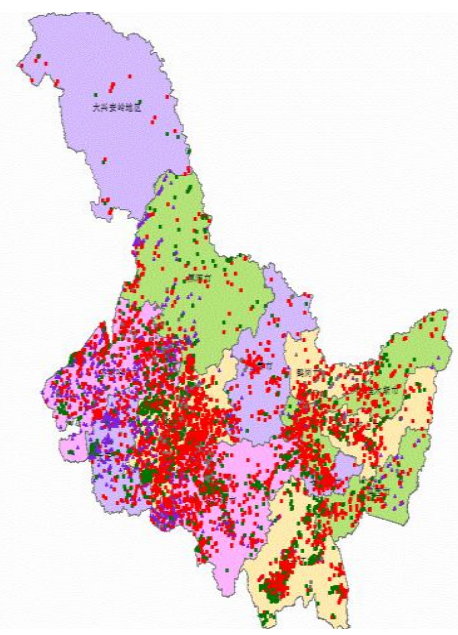

$c$. The map of the conflation result

Figure 5. The Figure of the Conflation Result 


\subsection{The Realization of Functions}

Heilongjiang animal husbandry information system contains the merging of business information modules and the merging of geographic information modules in the large-scale farms information monitoring system and the farming enterprises filing system.

The part of business information realizes the functions of farms reporting, system management, data management, bulk import, data reporting, query and statistic, information management, upcoming events, quarantine inspection certification, view of the farms, data statistics, and smart telephone terminals.

The part of geographic information realizes the following functions by ArcGIS. Firstly, the system has a variety of GIS-based property features, including zoom in, zoom out, pan, global map, backward, forward, map properties, magnifying glass, farm statistics, measurement and the display of Hawkeye. Secondly, the corresponding geographic information of large-scale farms and farming communities can be analyzed, inquired and counted, including the display of information, distribution of maintenance, rendering and inquiring and map resource.

\section{Conclusion}

This research applies the map conflation technology based on topological relation to the part of geographic information of the Heilongjiang animal husbandry information system, namely, merging the geographic information parts of large-scale farms information monitoring system and the farming enterprises filing system together. Using optimized "Spider code" and matching algorithm based on area overlay rate, it solves the map database conflation problem of two different sources while with the same geographic target in Heilongjiang. It not only improves the map accuracy and consistency, but also adds new space characteristics, and updates attribute information which associated with dataset spatial characteristics. The location of farms or farming communities which has been recorded or the location of all the farms or farming communities and the detailed information of the surroundings can be monitored visually. The personnel at all levels in the Animal Husbandry Bureau in Heilongjiang Province could share the information. It is conducive to promote the work of staff at all levels and provide decision support of data to the Animal Husbandry Bureau in Heilongjiang Province.

\section{Acknowledgements}

This research was supported by the National High-tech R\&D Program of China (863 Program) (2012AA101905) and the Key Technologies R\&D Program of Harbin (2014AB1BN035).

\section{References}

[1] Z. Yan, J. Tao and J. Xu, Research of Agricultural Modernization, vol. 33, (2012), pp. 13-18.

[2] Y. Feng, H. Chen and H. Peng, China Dairy, vol. 122, (2012), pp. 2-7.

[3] J. Zhou, P. Li, W. Meng, Y. Yin and Wei Li, Chinese Journal of Animal Science, vol. 48, (2012), pp. 21-24.

[4] Z. Li, W. Jian, Z. Qingdong and J. Wang, Transactions of the Chinese Society of Agricultural Engineering, vol. 22, (2006), pp. 39-43.

[5] W. Bai, R. Zhang, C. Shi, W. Wang and Y. Wang, Transactions of the Chinese Society of Agricultural Engineering, vol. 27, (2011), pp. 195-201.

[6] X. Xu, H. Zhang, L. Xi, X. Ma and J. Wang, Transactions of the Chinese Society of Agricultural Engineering, vol. 27, (2011), pp. 94-98.

[7] X. Tong and S. Deng, Geomatics and Information Science of Wuhan University, vol. 32, (2007), pp. 621-625.

[8] D. Li, J. Gong and Q. Zhang, Science of Surveying and Mapping, vol. 29, (2004), pp. 1-4. 
[9] L. Guo, "Theory and method research on multi-sources geo-spatial vector data fusion", PLA Information Engineering University, Zhengzhou, (2008).

[10] A. Saafeld, International Journal of Geographical Information Systems, vol. 2, (2008), pp. 217-228.

[11] D. He, J. Chen and J. Zhang, Journal of Jiangxi Normal University (Natural Sciences Edition), vol. 35, (2011), pp. 57-60.

[12] Z. Yang and S. Zhang, Digital Technology and Application, vol. 4, (2012), pp. 190-192.

[13] D. Susu and T. Xiaohua, Engineering of Surveying and Mapping, vol. 15, (2006), pp. 12-15.

[14] W. Tang, Y. Zhao, Y. Hao, J. Tang and W. Fan, Journal of System Simulation, vol. 21, (2009), pp. 1399-1402.

[15] X. Zhang and D. Yang, Journal of Jiangxi Normal University (Natural Sciences Edition), vol. 34, (2010), pp. $57-62$.

\section{Author}

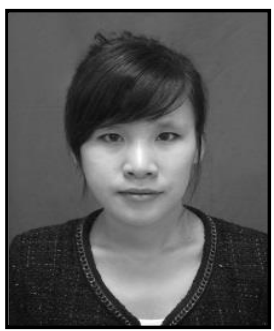

Yue Guo, (1990), female, M.S., mainly engaged in the design and research of application system based on GIS. 